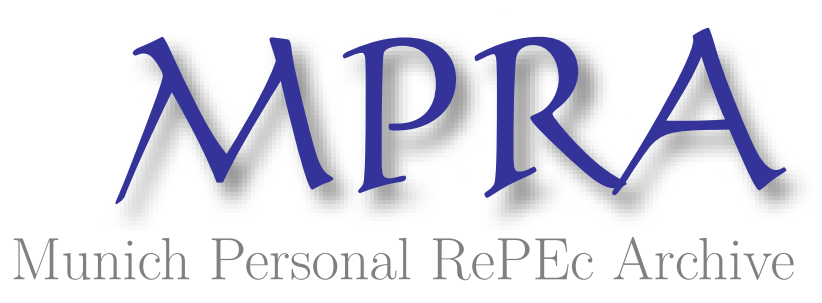

\title{
Persuasion, Spillovers, and Government Interventions
}

Li, Cheng and Xiao, Yancheng

2020

Online at https://mpra.ub.uni-muenchen.de/103500/

MPRA Paper No. 103500, posted 16 Oct 2020 04:25 UTC 


\title{
Persuasion, Spillovers, and Government Interventions
}

\author{
Cheng Li \& Yancheng Xiao*
}

August, 2020

\begin{abstract}
We develop a model of Bayesian persuasion with spillovers to investigate the impact of information production on optimal policy design. A sender produces information to persuade a receiver to take an action with external effects, and the government implements corrective subsidies and taxes to maximize social welfare. Subsidies to the sender's preferred action incentivize her to produce less information, while taxes motivating her to produce more. Such an informational effect impacts the receiver's decision and social welfare. We show that the optimal corrective subsidies and taxes may be different from the Pigouvian level. Most notably, the optimal policy is no government intervention when the spillover is positive and small.
\end{abstract}

Keywords: persuasion; spillover effects; externalities; Pigouvian taxes; subsidies; social welfare JEL: C7, D8, H2

${ }^{*}$ Li: Mississippi State University, Department of Finance and Economics, Mississippi State, MS 39762, USA. Email: cheng.li@msstate.edu. Xiao: School of Economics and Management, South China Normal University, Guangzhou, 510006, China. Email: yanchengxiao@m.scnu.edu.cn. 


\section{Introduction}

It is well known that many economic activities have external effects. By replacing its old fleet with more fuel-efficient aircrafts, an airline can reduce its carbon emissions. By loosening environmental regulations on coal mining, a government motivates the coal industry to generate more air pollution. Although the external effects of these actions are certain, the airline and the government may be uncertain about how these actions affect their payoffs. In particular, more fuel-efficient aircrafts are typically more costly to purchase and operate. Job-saving deregulation for the coal mining industry may hurt workers in the renewable energy industry. Therefore, to persuade the airline to buy newly designed aircrafts, manufacturers usually provide various information, such as reports about fuel efficiency and maintenance costs. To persuade the government to loosen environmental regulations, lobbyists often produce research on the economic benefits of job creation in the coal industry. Motivated by these examples, we develop a model of Bayesian persuasion with spillovers.

We adopt the Bayesian persuasion framework developed by Kamenica and Gentzkow (2011) to study the interaction between government intervention and information production. In the model, a sender produces information to persuade a receiver to take an action. The impact of the action on the receiver is uncertain, but the action certainly generates externalities on society. Since the receiver neglects the externalities of the action, his decision may lead to inefficient outcomes. Therefore, a policymaker can impose a subsidy or tax on the receiver to maximize social welfare.

A common solution to externalities is Pigouvian subsidies and taxes. ${ }^{1}$ According to Pigou (1920), the government should subsidize those who produce positive externalities by an amount equivalent to the external benefits and tax those who produce negative externalities in an amount equal to the external costs. Such a policy is indeed optimal in an environment where the receiver has exogenous information about his payoffs. In this paper, we consider an environment in which the sender endogenously produces information for the receiver and investigate the optimal

\footnotetext{
${ }^{1}$ Other solutions to externalities include Coasian bargaining (Coase 1960), permit markets for pollution rights (Arrow 1970), the folk theorem of repeated interactions (Fudenberg and Maskin 1986), and incentive mechanisms such as compensation mechanism (Varian 1994).
} 
value of corrective subsidies and taxes in such environment.

We make three important contributions to the literature. First, we find an informational effect of corrective subsidies and taxes. We show that corrective subsidies and taxes not only affect the decision-making of the receiver but also influence the sender's information production strategy. Specifically, subsidies reduce the cost of taking the sender's preferred action, making the receiver less hesitant to act. Therefore, the sender responds to greater subsidies by producing less information. Taxes, however, have the opposite informational effect as subsidies because taxes increase the cost of taking the sender's preferred action and make the receiver more skeptical. To our knowledge, we are the first to find such an informational effect of corrective subsidies and taxes.

Second, we show that the optimal value of corrective subsidies and taxes may be different from the Pigouvian level due to the informational effect illustrated above. In our model, corrective subsidies and taxes not only improve social welfare by internalizing externalities but also affect social welfare through the informational effect. Firstly, subsidies make it easier for the sender to persuade the receiver to take her preferred action and thus improve the sender's welfare. Secondly, subsidies incentivize the sender to produce less information, leading to less informed decisions and lower welfare for the receiver. Since taxes have the opposite informational effect as subsidies, taxes reduce the sender's welfare and increase the receiver's welfare. Then we show that the optimal subsidies and taxes may be different from the Pigouvian level. Most notably, when the sender's preferred action has small positive external effects, social welfare can be maximized without any subsidies. Our result is in contrast to the conventional wisdom that government interventions are necessary to solve the externalities problem and highlights the importance of considering endogenous information production for optimal policy design.

Finally, we apply our model to study fiscal federalism and show that decentralized decision-making can be optimal in the presence of interjurisdictional spillovers. We can think of the sender as a lobbying group and the receiver as a local government. The lobbying group wants to persuade the local government to implement a project with interjurisdictional spillovers. Similar to the novel result above, we show that decentralized decision-making is optimal when the positive interjurisdictional spillover is small. The intuition is that with small positive externalities, the cost of less in- 
formed decision-making dominates the benefit of centralization, so decentralization is optimal. Although previous studies have shown that decentralized policymaking can be optimal in the presence of interjurisdictional spillovers, the mechanisms driving their results are very different from ours. ${ }^{2}$ Therefore, our analysis provides a novel theory as to why decentralized decision making may be optimal in the presence of interjurisdictional spillovers.

\section{Model}

We consider a model of Bayesian persuasion with spillovers. There are two strategic players: Sender and Receiver. Sender wants to persuade Receiver to take an action, which affects the payoffs of Sender, Receiver and a non-strategic player called Bystander.

Receiver's action, denoted by $a$, is chosen from action space $A=\{0,1\}$. Receiver's payoff $u_{R}(a, \theta)$ depends on his own action $a \in A$ and an unknown state of the world $\theta \in \Theta=\{0,1\}:$

$$
u_{R}(a, \theta)=a(\theta-c)
$$

As shown in (1), Receiver receives a payoff of 0 from action $a=0$ regardless of the state of the world. When taking action $a=1$, Receiver incurs a cost of $c$ and receives a benefit that equals to the state of the world $\theta$. We assume $0<c<1$. Thus, Receiver prefers action $a=1$ to $a=0$ when $\theta=1$ and the opposite when $\theta=0$.

Sender and Bystander both have state-independent preferences. When Receiver takes action $a=0$, both receive a payoff of 0 . But when Receiver takes action $a=1$, Sender and Bystander receive a payoff of $\gamma_{1}$ and $\gamma_{2}$ respectively. We assume that $\gamma_{1}>0$ and thus Sender prefers action $a=1$ to action 0 regardless of the state of the

\footnotetext{
${ }^{2}$ The studies on fiscal federalism are interested in the optimal allocation of decision rights within the government (e.g. Oates 1972, Besley and Coate 2003, Ogawa and Wildasin 2009, Eichner and Runkel 2012, Fell and Kaffine 2014). Ogawa and Wildasin (2009) and Eichner and Runkel (2012) consider a model where jurisdictions are linked by a competitive market of capital, which produces emissions when it is used for production. In their model, a jurisdiction's choice of capital taxes is distorted by its incentives for tax competition and its inattention of interjurisdictional spillover effects. They show that both distortions could neutralize each other, making decentralized taxation optimal. Fell and Kaffine (2014) show that the results in Ogawa and Wildasin (2009) depend on two important assumptions and they may not hold in a more general model.
} 
world. The value of $\gamma_{2}$, however, can be either positive or negative, depending on the nature of action $a=1$.

We can think of Sender as Boeing, Receiver as an airline, and Bystander as the general public. Boeing prefers the airline to buy its newly designed aircrafts. The general public also prefers new planes because they produce fewer carbon emissions than the current fleet. Although the general public is certain about the emission reduction, the airline is uncertain whether the benefits of the new aircrafts outweigh their costs. Therefore, both $\gamma_{1}$ and $\gamma_{2}$ are positive. Similarly, we can consider Sender as the coal mining industry in West Virginia, Receiver as the state government and Bystander as the neighboring states. The coal mining industry prefers the state government to weaken environmental regulations. Although weaker environmental regulations may benefit the state economy, the state government is uncertain whether the benefits dominate the environmental costs. Pollutions caused by the weaker environmental regulations, however, certainly hurt the neighboring states. In this case, $\gamma_{1}$ is positive but $\gamma_{2}$ is negative.

The state of the world $\theta \in \Theta=\{0,1\}$ is ex-ante unknown to everyone. The common prior belief is that $\theta=1$ with probability $\mu_{0} \in(0,1)$ and $\theta=0$ with probability $1-\mu_{0}$. Following the Bayesian persuasion literature, we assume that Sender can influence Receiver's action by producing information about the state of the world $\theta$. Formally, Sender designs a costless signal, denoted by $\pi$, which is composed of two probability distributions $\pi(\cdot \mid \theta=1)$ and $\pi(\cdot \mid \theta=0)$ over a finite realization space $S$. Signal $\pi$ then generates a public realization $s \in S$. Based on signal $\pi$ and its realization $s \in S$, Receiver updates his belief about the state of the world according to the Bayes' rule:

$$
\mu \equiv \operatorname{Pr}(\theta=1 \mid s)=\frac{\mu_{0} \pi(s \mid \theta=1)}{\mu_{0} \pi(s \mid \theta=1)+\left(1-\mu_{0}\right) \pi(s \mid \theta=0)} .
$$

where $\mu$ represents the posterior belief of the state being $\theta=1$. Receiver then chooses the action that gives him the highest payoff based on $\mu$.

Bayesian persuasion models typically assume that Sender can control the signal design but not the signal realization. That is, Sender can control how evidence is produced (e.g. pharmaceutical companies control how clinical trials are conducted, aircraft manufacturers decide how fuel consumption is calculated), but she cannot forge evidence. Therefore, Sender is unable to control precisely what posterior beliefs will be generated, but she can control the distribution of posterior beliefs by 
designing signal $\pi$.

We use $\tau$ to denote the distribution of posterior beliefs induced by signal $\pi$. Distribution $\tau$ is Bayes-plausible if its expected value equals the prior belief $\mu_{0}$, i.e. $E[\tau]=\mu_{0}$. Kamenica and Gentzkow (2011) show that to derive Sender preferred signal, it is sufficient to derive the Bayes-plausible distribution of posterior beliefs preferred by Sender. Therefore, Sender's signal design problem is to choose $\tau$ to maximize her expected payoff subject to $E[\tau]=\mu$. In our model, maximizing Sender's expected payoff is equivalent to maximizing the probability of Receiver taking action $a=1$.

We define social welfare as the sum of the payoffs of Sender, Receiver and Bystander:

$$
W(a, \theta)=a\left(\theta-c+\gamma_{1}+\gamma_{2}\right) .
$$

By comparing the social welfare function and Receiver's utility function, we can see that Receiver's preferences are not perfectly aligned with the society. This is because Receiver neglects the spillover effects. We define $\gamma \equiv \gamma_{1}+\gamma_{2}$ and refer to $\gamma$ as the aggregate spillover effects of action $a=1$.

Lastly, the timing of the game is as follows. First, the government decides the amount of subsidy or tax to maximize social welfare. Second, Sender observes the subsidy or tax and design signal $\pi$. Third, Receiver observes the signal and its realization, updates his belief about the state of the world according to the Bayes' rule, and then chooses his action.

\section{Analysis}

In this section, we first show that the Pigouvian subsidies and taxes are optimal in a benchmark case when there is no information production by Sender. We then show how optimal subsidies and taxes may be different from the Pigouvian level when information is endogenously produced by Sender through Bayesian persuasion. 


\subsection{Benchmark: No Persuasion}

If Sender does not produce information about the state of the world $\theta$, Receiver makes decisions based on his prior belief $\mu_{0}$. In this case, his optimal action is:

$$
a\left(\mu_{0}\right)= \begin{cases}1 & \text { if } \mu_{0} \geq c \\ 0 & \text { otherwise }\end{cases}
$$

Meanwhile, the ex ante efficient action is

$$
a^{E E}\left(\mu_{0}\right)= \begin{cases}1 & \text { if } \mu_{0} \geq c-\gamma \\ 0 & \text { otherwise }\end{cases}
$$

Apparently, Pigouvian subsidies and taxes are necessary for efficient outcomes when there is no information production by Sender. When $\gamma>0$ and $c-\gamma<\mu_{0}<c$, the government can induce efficient outcomes by providing subsidy $t=\gamma$ (financed by a tax on Bystander) for action $a=1$. When $\gamma<0$ and $c<\mu_{0}<c-\gamma$, the government can induce efficient outcomes by taxing action $a=1$ in the amount of $T=|\gamma|$.

\subsection{Persuasion with Positive Spillovers}

Now we consider the case when the aggregate spillover is positive, i.e. $\gamma>0$. We focus on the prior beliefs for which it is ex ante necessary for government intervention:

$$
c-\gamma<\mu_{0}<c
$$

As shown in Section 3.1, if there is no information production, and (A1) holds, it is ex ante optimal to provide Pigouvian subsidies for action $a=1$. We are interested in whether the Pigouvian subsidies are still optimal for this range of prior beliefs when Sender produces information through Bayesian persuasion.

Suppose the government provides Receiver a subsidy of $t \geq 0$ for taking action $a=1$, which is financed by a tax on Bystander. ${ }^{3}$ We first pin down Sender's optimal signal given any subsidy and then derive the value of subsidy that maximizes social welfare.

\footnotetext{
${ }^{3}$ The qualitative results continue to hold if we assume that the subsidy is financed through a lump-sum tax on all the players in the model.
} 


\subsubsection{Optimal signal}

Given posterior belief $\mu$ and subsidy $t$, Receiver prefers action $a=1$ when

$$
\mu-c+t \geq 0 \Leftrightarrow \mu \geq c-t,
$$

where $c-t$ refers to Receiver's threshold of doubt.

Under Assumption (A1), $c-\mu_{0}$ is positive. When $t \geq c-\mu_{0}$, or $c-t \leq \mu_{0}$, the subsidy is sufficient to motivate Receiver to take action $a=1$ because his threshold of doubt is below the prior belief. Thus, Sender does not need to persuade Receiver.

When $t<c-\mu_{0}$, or $c-t>\mu_{0}$, the subsidy is insufficient, and Receiver's threshold of doubt is above the prior belief. That is, Receiver is ex ante against action $a=1$, and Sender must attempt to persuade. Therefore, Sender's optimal strategy is to design a signal that generates the following Bayes-plausible distribution of posterior beliefs:

$$
\tau^{*}=\left\{\begin{array}{cl}
0 & \text { with probability } 1-\frac{\mu_{0}}{c-t} \\
c-t & \text { with probability } \frac{\mu_{0}}{c-t}
\end{array}\right.
$$

Figure 1 shows that, by generating posterior beliefs 0 and $c-t$ while keeping the mean at $\mu_{0}$, Sender can achieve expected payoff that equals the red dotted line, which is strictly above her payoff from no persuasion (i.e. zero). The difference between the red dotted line and the horizontal axis represents Sender's gain from persuasion. As shown by Kamenica and Gentzkow (2011), the red dotted line (the concave closure of Sender's expected payoff) represents largest expected payoff that Sender can achieve given prior belief $\mu_{0}$. Therefore, the distribution of posterior beliefs in (7) is optimal for Sender. Such a distribution is also feasible because it is Bayes-plausible (i.e. its mean equals the prior belief $\mu_{0}$ ).

Sender can generate the distribution of posterior beliefs in (7) in many ways, one of which is committing to recommend action $a=1$ with the following probabilities:

$$
\operatorname{Pr}(\text { recommend } \mid \theta=0)=\frac{\mu_{0}(1-c+t)}{(c-t)\left(1-\mu_{0}\right)},
$$

and

$$
\operatorname{Pr}(\text { recommend } \mid \theta=1)=1 .
$$

If Sender does not recommend action $a=1$, Receiver is certain that $\theta=0$ and prefers action $a=0$. If Sender recommends action $a=1$, Receiver's posterior belief becomes 


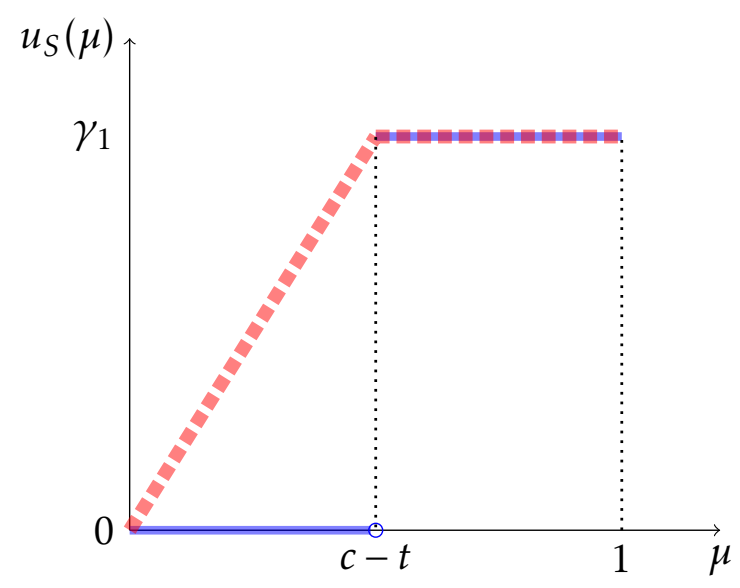

Figure 1: Sender's payoff as a function of the posterior belief (solid line) and its concave closure (dotted line).

$c-t$, which is his threshold of doubt. Although Receiver is still uncertain about the value of $\theta$ after receiving Sender's recommendation, the updated information is just enough to persuade him to take action $a=1$.

Sender's signal becomes less informative if Sender raises the probability of recommending action $a=1$ when the state of the world is $\theta=0$. Therefore, the larger the probability in equation (8), the less informative Sender's signal. It is straightforward to show that the probability in equation (8) is increasing in subsidy $t$. Therefore, Sender's optimal signal becomes less informative in response to greater subsidies. The intuition is that an increase in the subsidy reduces Receiver's threshold of doubt, which encourages Receiver to take action $a=1$.

To see why Sender's optimal signal becomes less informative when subsidies increase, we can also look at the distribution of posterior beliefs generated by Sender's optimal signal. As $t$ increases, Sender's optimal distribution of posterior beliefs in (7) becomes less "spread out" and thus corresponds to a less informative signal.

Proposition 1 When the aggregate spillover effect $\gamma$ is positive and the prior belief $\mu_{0}$ satisfies (A1), the informativeness of Sender's optimal signal is decreasing in subsidy $t$ if $t<c-\mu_{0}$.

Proposition 1 highlights an informational effect of corrective subsidies. In an environment where information is endogenously produced by Sender, corrective 
subsidies not only affect Receiver's decision-making but also influence Sender's information production. If the subsidy itself is insufficient to motivate Receiver to take action $a=1$, Sender designs a partially informative signal in equilibrium: she produces just enough information about the state of the world to maximize the probability that Receiver taking action $a=1$. In this case, greater subsidies encourage Receiver to act and reduce the difficulty to persuade Receiver. Therefore, Sender responds to greater subsidies by producing less information.

\subsubsection{Optimal subsidies}

Given Sender's optimal signal, we can derive the expected social welfare $E[W]$. When $t \geq c-\mu_{0}$, the subsidy is sufficiently large. Thus, Sender produces no information, and Receiver always chooses action $a=1$ in equilibrium. The equilibrium payoffs of Sender, Receiver and Bystander are $\gamma_{1}, \mu_{0}-c+t$ and $\gamma_{2}-t$ respectively. ${ }^{4}$ Social welfare is the sum of everyone's payoff and thus equals

$$
\mu_{0}-c+\gamma
$$

When $t<c-\mu_{0}$, Sender designs a signal that generates the following Bayesplausible distribution of posterior beliefs

$$
\tau=\left\{\begin{array}{cl}
0 & \text { with probability } 1-\frac{\mu_{0}}{c-t} \\
c-t & \text { with probability } \frac{\mu_{0}}{c-t}
\end{array}\right.
$$

If the posterior belief equals 0 , Receiver does not take action $a=1$ in equilibrium, giving everyone zero payoff. But if the posterior belief equals $c-t$, Receiver takes action $a=1$ in equilibrium. In this case, Sender's equilibrium payoff is $\gamma_{1}$, Receiver's equilibrium payoff is $(c-t)-c+t=0$ and Bystander's equilibrium payoff $\gamma_{2}-t$. Since posterior belief $c-t$ appears with probability $\mu_{0} /(c-t)$, the expected social welfare is

$$
E[W]=\underbrace{\frac{\mu_{0}}{c-t} \times \gamma_{1}}_{\text {Sender's payoff }}+\underbrace{\frac{\mu_{0}}{c-t} \times 0}_{\text {Receiver's payoff }}+\underbrace{\frac{\mu_{0}}{c-t} \times\left(\gamma_{2}-t\right)}_{\text {Bystander's payoff }}=\frac{\mu_{0}(\gamma-t)}{c-t}
$$

Equation (12) shows various welfare effects of the subsidy on each agent. First, Sender's payoff $\mu_{0} \gamma_{1} /(c-t)$ is increasing in subsidy $t$. Second, Receiver's payoff is

\footnotetext{
${ }^{4}$ Bystander's payoff is $\gamma_{2}-t$ because we assume that the subsidy to Receiver is financed by a tax on Bystander.
} 
zero. Although corrective subsidies raise Receiver's payoff from taking action $a=1$, they also incentivize Sender to produce less information, leading to less informed decisions by Receiver. That is, as subsidy rises, Sender becomes less likely to tell the truth when the state of the world $\theta=0$, which hurts Receiver. These two effects cancel each other, so Receiver's expected payoff remains at zero regardless of the value of the subsidy. Third, Bystander's payoff is $\mu_{0}\left(\gamma_{2}-t\right) /(c-t)$, which is increasing in $t$ if $\gamma_{2}>c$, and decreasing in $t$ otherwise. This is because the net effect of subsidies depends on the size of the spillover effect. On one hand, greater subsidies make Receiver more likely to take action $a=1$, benefiting Bystander through positive spillovers. On the other hand, greater subsidies lay more tax burden on Bystander. The following lemma summarizes the expected social welfare function.

Lemma 1 Given Sender's optimal signal, the expected social welfare is:

$$
E[W \mid t]= \begin{cases}\mu_{0}-c+\gamma & \text { when } t \geq c-\mu_{0} \\ \frac{\mu_{0}}{c-t}(\gamma-t) & \text { when } t<c-\mu_{0}\end{cases}
$$
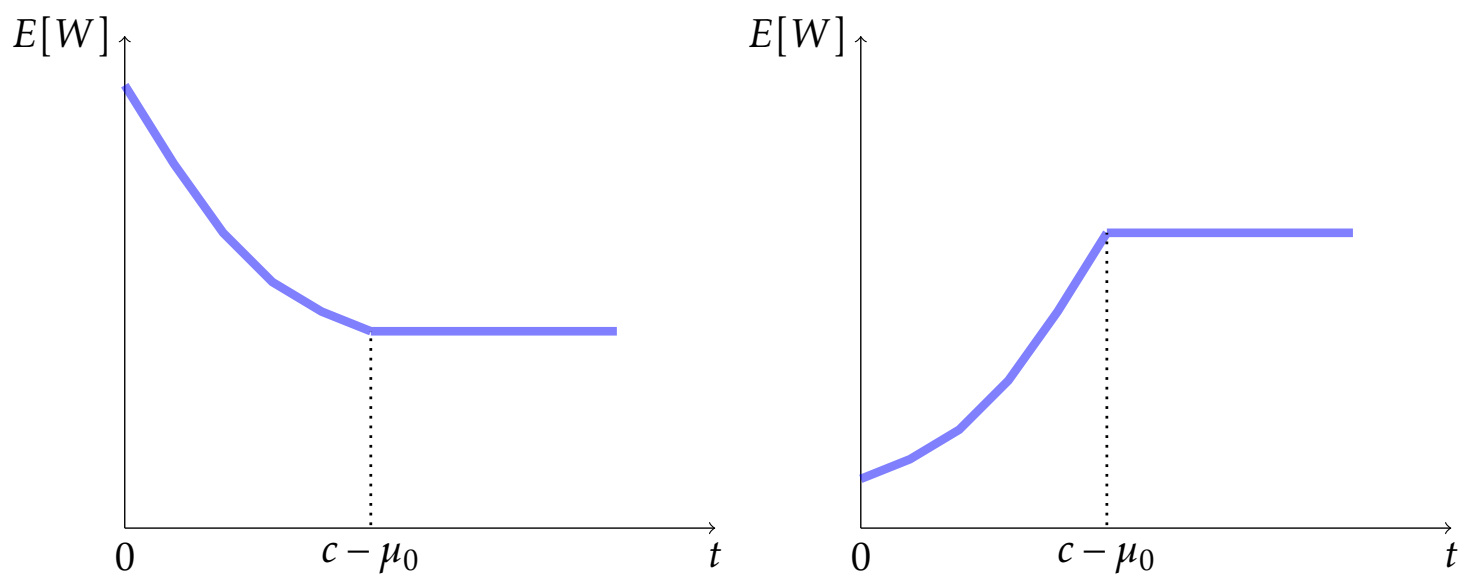

Figure 2: The expected social welfare as a function of subsidy $t$. The left figure shows the case when $\gamma<c$. The right figure shows the case when $\gamma>c$.

Figure 2 shows the expected social welfare as a function of subsidy $t$. Firstly, social welfare remains constant for any $t \geq c-\mu_{0}$. This is because when subsidies are sufficiently large, Receiver always take Sender's preferred action in equilibrium and social welfare is fixed at $\mu_{0}-c+\gamma$. 
Secondly, subsidies that are less than $c-\mu_{0}$ not only affect social welfare through the traditional channels of internalizing spillovers but also influence social welfare through the informational channels illustrated above. For relatively small externalities (when $0<\gamma<c$ ), the welfare costs associated with subsidies dominate the benefits, and the expected social welfare is decreasing in $t$. In this case, the expected social welfare is maximized when $t=0$, implying that no government intervention is optimal. For relatively large externalities (when $\gamma \geq c$ ), the benefits of subsidies dominate the welfare costs, so the expected social welfare is increasing in $t$. In this case, social welfare is maximized by any $t \geq c-\mu_{0}$, including the Pigouvian subsidy $t=\gamma$.

Proposition 2 When the aggregate spillover effect $\gamma$ is positive and the prior belief $\mu_{0}$ satisfies (A1), social welfare is maximized without subsidies if the spillover effect is small (i.e. $\gamma<c$ ). For greater spillover effects (i.e. $\gamma \geq c$ ), social welfare is maximized by subsidies that are no less than $c-\mu_{0}$, including the Pigouvian subsidy $t=\gamma$.

Although the Pigouvian subsidy is optimal in the benchmark case, it is no longer optimal when information is endogenously produced. After considering endogenous information production, we show that corrective subsidies not only internalize spillovers by changing Receiver's action but also affect Sender's information production strategy. Interestingly, social welfare can be maximized without subsidies when positive spillovers are small. This result contrasts the conventional wisdom that we need government interventions to internalize externalities. Therefore, our analysis has important policy implications: when designing policies to correct externalities, policymakers should not only understand the magnitude of spillovers but also be aware of the potential policy impact on endogenous information production.

\subsection{Negative Spillovers}

In this section, we consider the case when the aggregate spillover of action $a=1$ is negative, i.e. $\gamma<0$. Since $\gamma \equiv \gamma_{1}+\gamma_{2}$ and $\gamma_{1}$ is always positive, a negative $\gamma$ implies that $\gamma_{2}$ is negative and $\left|\gamma_{2}\right|>\gamma_{1} .{ }^{5}$ This is the case when Bystander's loss from action

\footnotetext{
${ }^{5}$ When $\gamma_{2}$ is negative and $\left|\gamma_{2}\right|<\gamma_{1}$, the aggregate spillover effect $\gamma$ is still positive and the analysis is similar to Section 3.2.
} 
$a=1$ outweighs Sender's gain from the action. Again, we focus on the range of prior beliefs for which it is ex ante necessary to have government interventions:

$$
c<\mu_{0}<c-\gamma
$$

As Section 3.1 shows, when (A2) is satisfied, Pigouvian taxes are necessary for ex ante efficiency. We are interested if such taxes continue to be optimal when information is endogenously produced by Sender.

Suppose the government imposes taxes of $T$ on action $a=1$ and the tax revenue is then transferred to Bystander. We are interested in the value of $T$ that maximizes social welfare. The derivation of Sender's optimal signal is similar to the analysis in Section 3.2 and is thus omitted. The following lemma summarizes Sender's optimal signal.

Lemma 2 When the government imposes a tax of $T$ on action $a=1$, Sender's optimal signal is as follows:

- when $T \geq 1-c$, Sender is indifferent between all signals.

- when $T<\mu_{0}-c$, Sender chooses a fully uninformative signal.

- when $\mu_{0}-c \leq T<1-c$, Sender chooses a partially informative signal that generates the following distribution of posterior beliefs:

$$
\tau=\left\{\begin{array}{cl}
0 & \text { with probability } 1-\frac{\mu_{0}}{c+T} \\
c+T & \text { with probability } \frac{\mu_{0}}{c+T}
\end{array}\right.
$$

Lemma 2 shows that if the tax is neither too small to be negligible nor too large to fully deter action $a=1$ (i.e. when $\mu_{0}-c \leq T<1-c$ ), Sender designs a partially informative signal in equilibrium. In this case, an increase in $T$ leads to a mean-preserving spread of the distribution of posterior beliefs generated by Sender's optimal signal. A mean-preserving spread of the distribution of posterior beliefs corresponds to a more informative signal. Therefore, greater taxes on Sender's preferred action induces Sender to produce more information. The next proposition summarizes such an informational benefit associated with greater taxes.

Proposition 3 When the aggregate spillover effect $\gamma$ is negative and the prior belief $\mu_{0}$ satisfies (A2), the informativeness of Sender's optimal signal is increasing in $\operatorname{tax} T$ if $\mu_{0}-c \leq T<1-c$. 
Taxes on action $a=1$ increases Receiver's threshold of doubt, making Receiver more hesitant to take the action. If the tax is above the Pigouvian level but insufficient to fully deter the action, it forces Sender to produce more information about the action. Such impact contrasts the informational effect of subsidies since subsidies make Receiver less hesitant to take Sender-preferred action and incentivize Sender to produce less information. Given Sender's optimal signal, we can calculate the expected social welfare and derive the optimal tax.

Lemma 3 Given Sender's optimal signal, the expected social welfare is

$$
E[W \mid T]=\left\{\begin{array}{cl}
\mu_{0}-c+\gamma & \text { when } T<\mu_{0}-c \\
\frac{\mu_{0}}{c+T}(\gamma+T) & \text { when } \mu_{0}-c \leq T<1-c \\
0 & \text { when } T \geq 1-c
\end{array}\right.
$$

Taxes greater than $1-c$ can deter Receiver from taking action $a=1$. Taxes smaller than $\mu_{0}-c$, however, can never deter Receiver from taking action $a=1$. In these cases, social welfare is independent of tax $T$. When $\mu_{0}-c \leq T<1-c$, corrective taxes not only internalizes the negative spillovers but also influence social welfare through two additional channels. First, as Proposition 3 shows, greater taxes can increase informativeness of the signal, leading to more informed decision-making and higher payoffs for Receiver. Second, greater taxes reduce Sender's payoff by lowering the probability that Receiver takes Sender's preferred action. it is straightforward to show that the welfare gain of Receiver and Bystander dominates Sender's welfare loss. Therefore, corrective taxes have a net positive effect on social welfare when $\mu_{0}-c \leq T<1-c$.

Figure 3 shows that social welfare is increasing in tax $T$ when $\mu_{0}-c \leq T<1-c$. When the spillovers are small (i.e. $|\gamma|<1-c$ ), corrective taxes can lead to positive social welfare and the optimal tax is $T=1-c$. When the spillovers are relatively large (i.e. $|\gamma| \geq 1-c$ ), the greatest feasible social welfare is zero and any tax no less than $1-c$ is optimal.

Proposition 4 When the aggregate spillover effect $\gamma$ is negative and the prior belief $\mu_{0}$ satisfies (A2), the optimal tax T equals $1-c$ if the spillover effect is small $(i . e .|\gamma|<1-c)$. When the spillover effect takes greater values (i.e. when $|\gamma| \geq 1-c$ ), the optimal tax is any $T$ no less than $1-c$. 

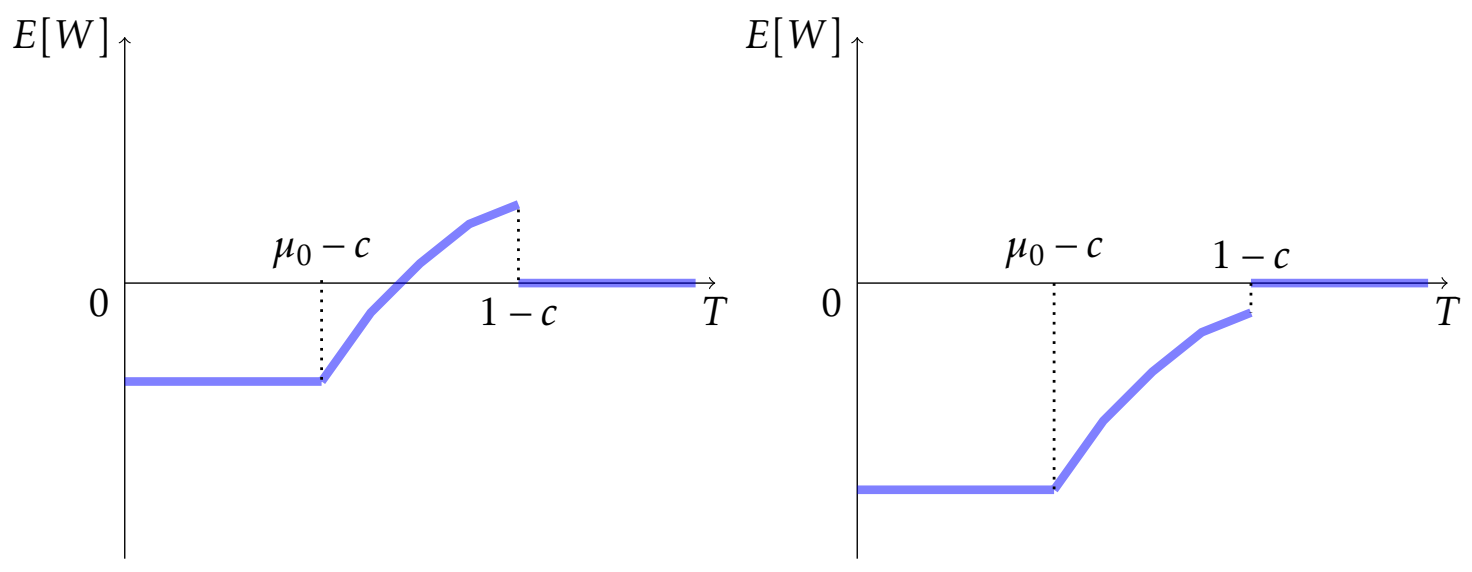

Figure 3: The expected social welfare as a function of $\operatorname{tax} T$. The left figure shows the case when $|\gamma|<1-c$ and the right figure shows the case when $|\gamma| \geq 1-c$.

For negative spillovers, corrective taxes are still necessary for efficient outcomes when we consider endogenous information production. However, the optimal value of corrective taxes may be different from the Pigouvian level due to the informational effect of corrective taxes. When the externalities are small (i.e. when $|\gamma|<1-c$ ), the optimal value of the tax is $1-c$, which is greater than the Pigouvian level. When the externalities are large (i.e. when $|\gamma| \geq 1-c$ ), any taxes greater than $1-c$ are optimal, including the Pigouvian $\operatorname{tax} T=|\gamma|$.

\section{Application: Interjurisdictional Spillovers}

In this section, we apply our model to compare centralized and decentralized decision-making when local decision making leads to interjurisdictional spillovers. The literature on fiscal federalism has analyzed this problem from various aspects (e.g. Oates 1972, Besley and Coate 2003, Ogawa and Wildasin 2009, Eichner and Runkel 2012, Fell and Kaffine 2014). We provide a novel perspective to investigate how the allocation of decision rights within the government affects special interest group's incentives to produce information. ${ }^{6}$

\footnotetext{
${ }^{6}$ Although it is well documented that special interest group often produce information to influence policy decisions (Austen-Smith and Wright 1992, Bennedsen and Feldmann 2006, Dahm and Porteiro 2008, Cotton 2012, Cotton and Dellis 2016), Li (2020) is the only paper that takes into informational lobbying when comparing centralized and decentralized decision-making. His paper, however,
} 
We can think of Sender as a lobbying group, Receiver as a local government, and Bystander as other jurisdictions in the nation. Action $a=1$ represents a local project that produces externalities to other jurisdictions. The lobbying group always prefers the local government to implement the project. The local government, however, is uncertain how the project affects the jurisdiction. To persuade the local government to implement the project, the lobbying group produces information about the project. We want to find the socially optimal allocation of authority: should we allow the local government to decide whether to implement the project or should we have a central government to make the decision?

A common assumption in the fiscal federalism literature is that the central government cares about social welfare while the local government only cares about the welfare in its jurisdiction. Therefore, under centralization, the central government internalizes spillovers and chooses action $a=1$ when:

$$
\mu-c+\gamma \geq 0 \Leftrightarrow \mu \geq c-\gamma
$$

Under decentralization, however, the local government chooses action $a=1$ when:

$$
\mu-c \geq 0 \Leftrightarrow \mu \geq c .
$$

Equations (15) and (16) show that only the central government considers the spillover effect. The central government acts as someone who receives a Pigouvian subsidy $t=\gamma$ when $\gamma>0$ and pays a Pigouvian tax $T=|\gamma|$ when $\gamma<0$. The local government, however, acts as someone who receives no subsidy and pays no tax. Therefore, to compare social welfare under centralization and decentralization, we just need to understand whether Pigouvian subsidies and taxes improve social welfare.

Figure 2 shows that for positive spillovers, Pigouvian subsidies $t=\gamma$ lead to higher social welfare than $t=0$ when $\gamma<c$ but the opposite when $\gamma \geq c$. This implies that decentralization is optimal for small positive interjurisdictional spillovers. The reasoning is similar to the cost-benefit analysis highlighted in Section 3.2. The central government considers positive spillovers and thus is more willing to implement the project than the local government. The lobbying group anticipates the does not consider interjurisdictional spillovers. Bordignon, Colombo and Galmarini (2008) and Lima, Moreira and Verdier (2017) consider monetary lobbying when comparing centralized and decentralized decision-making, but they do not consider information production by interest groups. 
raised willingness and produces less information about the project to the central government. Therefore, when the spillovers are small, the welfare loss associated with such an informational effect dominates the benefits of centralization, making decentralization optimal.

Figure 3 shows that for negative spillovers, a tax $T=|\gamma|$ always lead to greater social welfare than $T=0 .^{7}$ Therefore, centralization is always optimal for negative interjurisdictional spillovers. The next proposition summarizes the findings above.

Proposition 5 For positive interjurisdictional spillovers and prior beliefs specified in (A1), decentralization is optimal when $\gamma<c$ and centralization is optimal when $\gamma \geq c$. For negative interjurisdictional spillovers and prior beliefs specified in (A2), centralization is always optimal.

Our analysis highlights a novel informational effect of centralized decisionmaking: when interjurisdictional spillovers are positive and small, centralized decision-making leads to less information produced by the lobbying group and less informed decision-making by the government. More importantly, the welfare loss associated with less informed decision-making dominates the benefits of centralization. Therefore, our analysis provides a novel theory to explain why decentralized decision-making could be optimal with the presence of interjurisdictional spillovers.

\section{Conclusions}

In this paper, we incorporate endogenous information production to study optimal policy design against externalities. We find a novel informational channel through which corrective subsidies and taxes affect social welfare. We find three important results. First, we show that subsidies to the sender's preferred action incentivize her to produce less information, while taxes motivating her to produce more. Second, we also show that the optimal value of subsidies and taxes may be different from the Pigouvian level. Most notably, social welfare is maximized without any subsidies when the spillover is positive and small. Third, we apply the model to investigate the allocation of authorization and find that decentralization is optimal when interjurisdictional spillover is positive and small.

\footnotetext{
${ }^{7}$ Note that $|\gamma|$ is larger than $\mu_{0}-c$ because of assumption A2.
} 
Our analysis highlights the importance of considering endogenous information production to study interventions to correct externalities. When policymakers design corrective subsidies and taxes, they should not only understand the magnitude of spillovers but also be aware of the potential policy impact on endogenous information production.

\section{References}

Arrow, J. Kenneth. 1970. The organization of economic activity: issues pertinent to the choice of market versus nonmarket allocation. Chicago: Markham.

Austen-Smith, David, and John R. Wright. 1992. "Competitive lobbying for a legislator's vote." Social Choice and Welfare, 9(3): 229-257.

Bennedsen, Morten, and Sven E. Feldmann. 2006. "Informational Lobbying and Political Contributions." Journal of Public Economics, 90: 631-656.

Besley, Timothy, and Stephen Coate. 2003. "Centralized versus Decentralized Provision of Local Public Goods: A Political Economy Approach." Journal of Public Economics, 87(12): 2611-2637.

Bordignon, Massimo, Luca Colombo, and Umberto Galmarini. 2008. "Fiscal federalism and lobbying." Journal of Public Economics, 92: 2288-2301.

Coase, Ronald H. 1960. "The Problem of Social Cost." Journal of Law and Economics, 3: $1-44$.

Cotton, Christopher. 2012. "Pay-to-play Politics: Informational Lobbying and Contribution Limits When Money Buys Access." Journal of Public Economics, 96: 369386.

Cotton, Christopher S., and Arnaud Dellis. 2016. "Informational Lobbying and Agenda Distortion." Journal of Law, Economics and Organization, 32(4): 762-793.

Dahm, Matthias, and Nicolas Porteiro. 2008. "Side Effects of Campaign Finance Reform." Journal of the European Economic Association, 6(5): 1057-1077. 
Eichner, Thomas, and Marco Runkel. 2012. "Interjurisdictional Spillovers, Decentralized Policymaking, and the Elasticity of Capital Supply." American Economic Review, 102(5): 2349-2357.

Fell, Harrison, and Daniel T. Kaffine. 2014. "Can decentralized planning really achieve first-best in the presence of environmental spillovers?" Journal of Environmental Economics and Management, 68: 46-53.

Fudenberg, Drew, and Eric Maskin. 1986. "The Folk Theorem in Repeated Games with Discounting or with Incomplete Information." Econometrica: Journal of the Econometric Society, 533-554.

Kamenica, Emir, and Matthew Gentzkow. 2011. "Bayesian Persuasion." American Economic Review, 101(6): 2590-2615.

Li, Cheng. 2020. "Centralized Policymaking and Informational Lobbying." Social Choice and Welfare, 54: 527-557.

Lima, Rafael Costa, Humberto Moreira, and Thierry Verdier. 2017. "Centralized Decision Making and Informed Lobbying." American Economic Journal: Microeconomics, 9(4): 324-355.

Oates, Wallace E. 1972. Fiscal Federalism. Harcourt Brace Jovanovich, New York.

Ogawa, Hikaru, and David E. Wildasin. 2009. "Think Locally, Act Locally: Spillovers, Spillbacks, and Efficient Decentralized Policymaking." American Economic Review, 99(4): 1206-1217.

Pigou, Arthur C. 1920. The Economics of Welfare. London: Macmillan and Co.

Varian, Hal R. 1994. "A solution to the problem of externalities when agents are well-informed." The American Economic Review, 1278-1293. 


\section{Appendix}

\section{Proof of Lemma 2}

Lemma 2 When the government imposes a tax of $T$ on action $a=1$, Sender's optimal signal is as follows:

- when $T \geq 1-c$, Sender is indifferent between all signals.

- when $T<\mu_{0}-c$, Sender chooses a fully uninformative signal.

- when $\mu_{0}-c \leq T<1-c$, Sender chooses a partially informative signal that generates the following distribution of posterior beliefs:

$$
\tau=\left\{\begin{array}{cl}
0 & \text { with probability } 1-\frac{\mu_{0}}{c+T} \\
c+T & \text { with probability } \frac{\mu_{0}}{c+T}
\end{array}\right.
$$

Given posterior belief $\mu$ and tax $T$, Receiver takes action $a=1$ when

$$
\mu-c-T \geq 0 \Leftrightarrow \mu \geq c+T,
$$

where $c+T$ is Receiver's threshold of doubt.

When $T \geq 1-c$, Receiver's threshold of doubt is larger than one, meaning that Receiver never takes action $a=1$ in equilibrium. In this case, Sender is indifferent between all the information production strategies.

When $0<T<1-c$, Receiver's threshold of doubt is between zero and one. In this case, Receiver's action depends on whether the prior belief is greater than the threshold of doubt, while Sender decides whether to produce information accordingly. Specifically, when $T<\mu_{0}-c$, the tax is relatively small, and the prior belief is still above Receiver's threshold of doubt (i.e. $\mu_{0}>c+T$ ). In this case, Receiver is ex ante in favor of action $a=1$, and thus Sender can ensure that Receiver chooses action $a=1$ by producing no information. When $\mu_{0}-c \leq T<1-c$, the tax is sufficiently large, and Receiver's prior belief is below the threshold of doubt (i.e. $\mu_{0} \leq c+T$ ). The receiver is ex ante against the action $a=1$ because of the tax. In this case, Sender has an incentive to produce information to persuade Receiver. Similar to the situation under positive spillover, Sender has a uniquely optimal signal that generates the 
following distribution of posterior beliefs

$$
\tau=\left\{\begin{array}{cl}
0 & \text { with probability } 1-\frac{\mu_{0}}{c+T} \\
c+T & \text { with probability } \frac{\mu_{0}}{c+T}
\end{array}\right.
$$

\section{Proof of Lemma 3}

Lemma 3 Given Sender's optimal signal, the expected social welfare is

$$
E[W \mid T]=\left\{\begin{array}{cl}
\mu_{0}-c+\gamma & \text { when } T<\mu_{0}-c \\
\frac{\mu_{0}}{c+T}(\gamma+T) & \text { when } \mu_{0}-c \leq T<1-c \\
0 & \text { when } T \geq 1-c
\end{array}\right.
$$

When $T<\mu_{0}-c$, Sender produces no information and Receiver chooses action $a=1$ in equilibrium. The sender's equilibrium payoff is $\gamma_{1}$, Receiver's equilibrium payoff is $\mu_{0}-c-T$ and Bystander's equilibrium payoff $\gamma_{2}+T$. Social welfare is thus

$$
\mu_{0}-c+\gamma
$$

When $\mu_{0}-c \leq T<1-c$, Sender designs a signal that generates the following Bayes-plausible distribution of posterior beliefs

$$
\tau=\left\{\begin{array}{cl}
0 & \text { with probability } 1-\frac{\mu_{0}}{c+T} \\
c+T & \text { with probability } \frac{\mu_{0}}{c+T}
\end{array}\right.
$$

When the posterior belief equals 0 , Receiver does not take action $a=1$ in equilibrium, giving everyone zero payoff. In this case, social welfare is zero. When the posterior belief equals $c+T$, Receiver takes action $a=1$ in equilibrium. The sender's equilibrium payoff is $\gamma_{1}$, Receiver's equilibrium payoff is $(c+T)-c-T=0$ and Bystander's equilibrium payoff $\gamma_{2}+T$. In this case, social welfare equals to $\gamma+T$. Therefore, the expected social welfare given Sender's optimal signal is

$$
\left(1-\frac{\mu_{0}}{c+T}\right) \times 0+\frac{\mu_{0}}{c+T}(\gamma+T)=\frac{\mu_{0}}{c+T}(\gamma+T) .
$$

Lastly, when $T \geq 1-c$, Receiver never takes action $a=1$, and everyone gets zero payoff. 